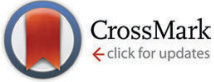

Cite this: Phys. Chem. Chem. Phys., 2016, 18, 17655

Received 21st April 2016, Accepted 7th June 2016

DOI: $10.1039 / \mathrm{c} 6 \mathrm{cp} 02678 \mathrm{~h}$

www.rsc.org/pccp

\section{Intersystem crossing rates of single perylene molecules in ortho-dichlorobenzene $\dagger$}

\author{
Nico R. Verhart, ${ }^{a}$ Pedro Navarro, ${ }^{b}$ Sanli Faez ${ }^{c}$ and Michel Orrit*a \\ Triplet states can be interesting for optical switching of molecular fluorescence as well as quantum \\ experiments relying on the manipulation of spin states. However, the ground state of molecules is \\ usually a singlet state. It is therefore interesting to study the intersystem crossing (ISC) rates between \\ singlet and triplet states. We have measured the autocorrelation function of the fluorescence from \\ single perylene molecules in an ortho-dichlorobenzene host matrix at cryogenic temperatures (1.3 K). \\ We observed two time scales in the autocorrelation function corresponding to intersystem crossing to \\ two indistinguishable triplet states $\left(T_{X}\right.$ and $\left.T_{Y}\right)$ and a third triplet state $\left(T_{Z}\right)$. By studying the power \\ dependence of the correlation times and contrasts in the autocorrelation functions of single molecules, \\ we determine the ISC rates of perylene for the first time.
}

\section{Introduction}

Intersystem crossing (ISC) to triplet states can severely limit the number of fluorescence photons that one can get from a fluorophore. Hence in order to be able to detect a single fluorophore by means of its fluorescence the ISC rates to the dark triplet states should not be too large (Table 3 shows the ISC rates of some commonly used fluorophores). On the other hand the triplet states themselves may be of interest: they could potentially be utilized to develop an all-optical single-molecule switch because of their relatively long lifetime ${ }^{1}$ or they could be useful in experiments that rely on the manipulation of spin, e.g. in experiments such as optically detected magnetic resonance (ODMR) in single molecules. ${ }^{2-5}$ In either case it is important to know the ISC rates. In this paper we determine the ISC rates of single perylene molecules by studying their fluorescence autocorrelation functions, a method that was successfully used in the past to determine the ISC rates of pentacene, ${ }^{6-8}$ terrylene, ${ }^{9-14}$ dibenzanthanthrene $\mathrm{e}^{15-17}$ terrylenediimide $^{18}$ and several perylene bisimide derivatives. ${ }^{19,20}$

Previously, measurements have been done on single perylene molecules in the host matrix polyethylene, ${ }^{21} n$-nonane ${ }^{22}$ and biphenyl. ${ }^{23}$ Attempts to measure single perylene molecules in anthracene were unsuccessful ${ }^{23}$ and measurements of terrylene in anthracene showed very low fluorescence intensities at saturation. ${ }^{11-13}$ This quenching of the fluorescence has been

\footnotetext{
${ }^{a}$ Huygens-Kamerling Onnes Laboratory, Leiden University, PO Box 9504, 2300 RA Leiden, The Netherlands.E-mail: orrit@physics.leidenuniv.nl

${ }^{b}$ National Autonomous University of Mexico, Mexico City, Mexico

${ }^{c}$ Nanophotonics, Debye Institute for Nanomaterial Science, Utrecht University, 3508 TA Utrecht, The Netherlands

$\dagger$ Electronic supplementary information (ESI) available. See DOI: 10.1039/c6cp02678h
}

attributed to a very efficient ISC mediated by the triplet level of the anthracene host which lies in between the excited singlet states and the triplet states of the guest molecules perylene and terrylene. It involves a two-step process consisting of a relaxation from the excited singlet state of the guest to the triplet state of the host followed by a transfer to the triplet state of the guest through a Dexter process (i.e. $\mathrm{S}_{1} \otimes \mathrm{S}_{0 \mathrm{~h}} \rightarrow \mathrm{S}_{0} \otimes \mathrm{T}_{1 \mathrm{~h}} \rightarrow \mathrm{T}_{1} \otimes \mathrm{S}_{0 \mathrm{~h}}$, where the subscript $\mathrm{h}$ is used to indicate the host). ${ }^{11-13}$ While the guest molecule resides in the long-lived triplet state it does not fluoresce as this would involve radiative transitions between the singlet states. In the case of perylene in anthracene Walla et al. were unable to observe single-molecule fluorescence while the bulk phosphorescence was rather bright. ${ }^{23}$ This was later attributed to a very efficient host-mediated intersystem crossing. ${ }^{11-13}$ Hence not every host matrix can be used to measure single perylene molecules.

Here we use a host matrix of 1,2-dichlorobenzene or orthodichlorobenzene $(o \mathrm{DCB})$, see Fig. 1. The singlet-singlet transition energy of $o$ DCB lies around $3.64 \times 10^{4} \mathrm{~cm}^{-1}$ (wavelength $275 \mathrm{~nm})^{24,25}$ and the singlet-triplet transition energy around $2.86 \times 10^{4} \mathrm{~cm}^{-1}$ (wavelength $\sim 350 \mathrm{~nm}$ ), well above the energy levels of perylene and terrylene. Therefore no host-mediated ISC is expected (of course the regular ISC is still present). Indeed $o$ DCB was successfully used as a host matrix for single-molecule measurements on terrylene. ${ }^{26}$ From bulk spectroscopy experiments on perylene in $o \mathrm{DCB}$ at $1.5 \mathrm{~K}$ the wavelength of the zero-phonon line was determined to be around $446 \pm 2 \mathrm{~nm}$ and some preliminary single-molecule measurements were done. ${ }^{27}$

Finally we briefly comment on the structural properties of the host. $o$ DCB has a melting point of $-18{ }^{\circ} \mathrm{C}$. Upon solidification $o$ DCB can form a crystal with monoclinic unit cell, ${ }^{28}$ however not much is known about the different polymorphs of $o$ DCB. For paradichlorobenzene, an isomer of $o \mathrm{DCB}$, several polymorphs exist. 

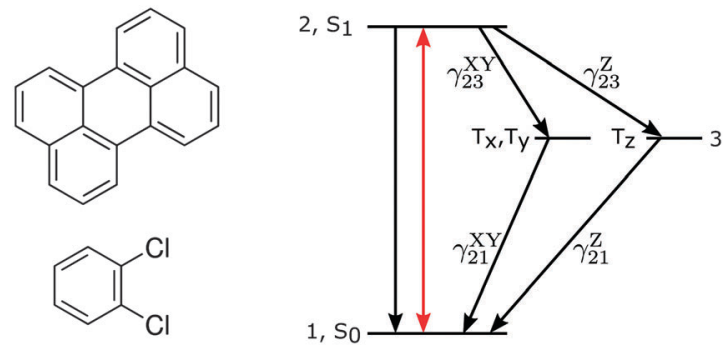

Fig. 1 Left: Structure formulas of the guest molecule perylene (top) and the host molecule ortho-dichlorobenzene (bottom). Right: A Jablonski diagram illustrating the relevant eigenstates of perylene and the transition rates between them. The rates involving the triplet states $T_{X}$ and $T_{Y}$ remain indistinguishable in the experiments presented in this paper.

\section{Experimental}

\subsection{Setup}

The setup consists of a low-temperature confocal microscope. The sample and microscope objective (Edmund Optics, 0.85NA) are mounted inside a flow cryostat (Janis, Model SVT-200-5) which can be cooled to about $1.3 \mathrm{~K}$. A tunable single-frequency diode laser (Toptica Photonics, DL Pro HP, grating-stabilized single-mode tunable diode laser) with a wavelength around $447 \mathrm{~nm}$ is used to excite the perylene molecules. The excitation light is passed through a clean-up filter (Chroma, ET440/20x) to remove undesired long-waved components. The microscope objective focuses excitation light on the sample and collects fluorescence from the sample. The collected Stokes-shifted fluorescence is separated from the excitation light by a dichroic beamsplitter (Semrock, FF458-Di02-25x36), filtered from residual laser light by a long-pass filter (Chroma, ET460lp) and passed through a spatial filter to reject light not originating from the focus. The filtered fluorescence is detected by an avalanche photo-diode (Excelitas, SPCM-AQRH-16).

\subsection{Sample preparation}

Samples were made by filling rectangular capillaries (VitroCom, inner dimensions $50 \mu \mathrm{m}$ by $500 \mu \mathrm{m}$, wall thickness $35 \mu \mathrm{m}$, VitroTubes 5005, borosilicate) with a solution of perylene (Sigma-Aldrich, sublimed grade, purity $\geq 99.5 \%, 394475$ ) in $o$ DCB (Sigma-Aldrich, CHROMASOLV, 99\%, 270 598). The fluid was sucked into the capillaries by capillary action. Typically solutions with a concentration in the range of 1-150 nM where found to be convenient. The capillaries where glued to a glass coverslip (Agar Scientific, $19 \mathrm{~mm}, \# 1.5$ ) and sealed at the ends at the same time using epoxy (Griffon, Combi, fast transparent 2-component epoxy glue, 6304033).

\section{Results and discussion}

Fig. 2 shows an excitation spectrum of the zero-phonon line of a single perylene molecule. Narrow Lorentzian zero-phonon lines were observed. As shown in Fig. 2, the power dependence of the FWHM and the peak fluorescence intensity of the excitation spectrum was studied. By extrapolating the results to low excitation
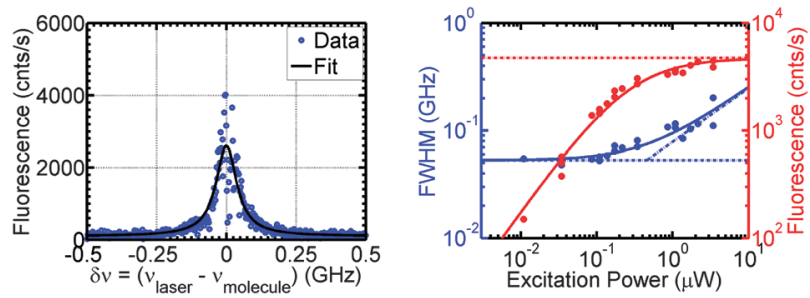

Fig. 2 Left: An excitation spectrum of the ZPL. For this specific spectrum, recorded near saturation ( $P_{\text {exc. }}=0.34 \mu \mathrm{W}$ ), the FWHM is $90 \pm 2 \mathrm{MHz}$ and the peak fluorescence intensity is $2.50 \pm 0.04 \mathrm{kcnts} \mathrm{s}^{-1}$. The additional noise close to the resonance is due to the ISC, similar to what was observed for perylene in $n$-nonane. ${ }^{22}$ Right: The power dependence of the line width (blue) and fluorescence intensity (red). The dots are the data, the solid curves are fits. Extrapolation to low powers allows us to determine the homogeneous line width of $53 \pm 3 \mathrm{MHz}$, while extrapolation to high power allows us to determine the maximum fluorescence rate of $4.7 \pm 0.3 \mathrm{kcnts} \mathrm{s}^{-1}$.

powers the homogeneous line width was determined to be $53 \pm 3 \mathrm{MHz}$. The somewhat low fluorescence intensity at saturation of about $4.7 \pm 0.3 \mathrm{kcnts} \mathrm{s}^{-1}$ (comparable to what was observed for perylene in biphenyl ${ }^{23}$ ) is probably due to a combination of the reduced quantum efficiency of the detector for blue light and the ISC of the molecule as discussed below. Most of the perylene molecules ( $\geq 90 \%$ ) show some spectral diffusion, often consisting of discrete jumps. However molecules could be found for which the spectral diffusion is slow (i.e. one jump or so on timescales of tens of seconds up to many hours).

The time trace of the fluorescence from a single molecule shown in Fig. 3 shows clear blinking behavior. As the host matrix is frozen, translational and rotational diffusion can be excluded and we attribute this blinking behavior to the ISC: while the molecule resides in one of the relatively long-lived triplet states no radiative transitions from the excited singlet state to the ground state can take place, hence ISC causes interruptions of the fluorescence. To analyze this data further we calculated the autocorrelation function $G^{(2)}(\tau)=\langle I(t) I(t+\tau)\rangle_{t} /\langle I\rangle_{t}^{2}$ shown in Fig. 3. The autocorrelation function shows two decaying components.

We attribute these two components to the transitions to and from the indistinguishable states $T_{X}$ and $T_{Y}$, jointly referred to as $\mathrm{T}_{\mathrm{XY}}$, and a third state $\mathrm{T}_{\mathrm{Z}}$, as illustrated in Fig. 1. As was pointed out in ref. 15 for ISC rates much smaller than fluorescenceand dephasing rates and two practically indistinguishable triplet states, the five-level optical Bloch equations can be replaced by a simpler three level rate model with a fluorescent state consisting of the singlet manifold (S) and two dark states consisting of the triplet states $\left(\mathrm{T}_{\mathrm{XY}}\right.$ and $\left.\mathrm{T}_{\mathrm{Z}}\right)$. The effective transition rates from the fluorescent state to the dark states are $k_{23}^{\mathrm{XY}}=\gamma_{23}^{\mathrm{XY}} \tilde{\rho}_{22}$ and

$\$$ It is difficult to completely exclude spectral diffusion from being responsible for the blinking behavior, however we note that slow spectral diffusion is unlikely because we select only those molecules that appear spectrally stable. Regarding the fast spectral diffusion and/or dephasing we note that the observed homogeneous linewidths have a magnitude comparable to the lifetime-limited linewidths, as explained at the end of this section. It is therefore unlikely that the observed intensity modulations are caused by spectral diffusion only. 

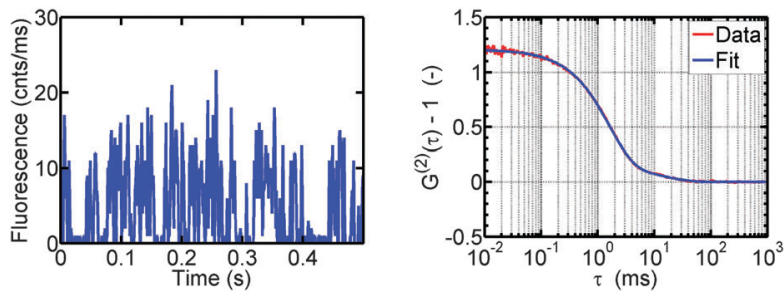

Fig. 3 Left: A fluorescence time trace from a single perylene molecule, showing blinking due to ISC. Right: An autocorrelation function of the fluorescence from a single perylene molecule (red curve) fitted by a bi-exponential decay. Two time scales can be observed: one around $1.60 \pm 0.02 \mathrm{~ms}$ and one around $16 \pm 1 \mathrm{~ms}$.

$k_{23}^{\mathrm{Z}}=\gamma_{23}^{\mathrm{Z}} \tilde{\rho}_{22}$. Here $\tilde{\rho}_{22}$ may be interpreted as the steady-state excited-state population given that the molecule is not in the triplet state. The transition rates from the dark states to the fluorescent states are $\gamma_{31}^{\mathrm{XY}}$ and $\gamma_{31}^{\mathrm{Z}}$. Within this rate model the autocorrelation function of the fluorescence is given by: ${ }^{15}$

$$
G^{(2)}(\tau)=\frac{p(\mathbf{S}, \tau \mid \mathbf{S}, 0)}{p_{0}(\mathbf{S})}=1+C_{+} \exp \left(-\lambda_{+} \tau\right)+C_{-} \exp \left(-\lambda_{-} \tau\right)
$$

with $p(\mathrm{~S}, \tau \mid \mathrm{S}, 0)$ the probability of the molecule being in the singlet manifold $\mathrm{S}$ at time $\tau$ given it was in the singlet manifold at time zero and $p_{0}(\mathrm{~S})$ the steady-state probability of being in the singlet manifold. The decay rates $\lambda_{ \pm}$and contrasts $C_{ \pm}$are given by: ${ }^{15} \S$

$$
\begin{gathered}
\lambda_{ \pm}=\frac{1}{2}(\Sigma \pm D) \\
C_{ \pm}=\frac{\lambda_{\mp}\left(\gamma_{31}^{\mathrm{XY}}-\lambda_{ \pm}\right)\left(\gamma_{31}^{\mathrm{Z}}-\lambda_{ \pm}\right)}{\gamma_{31}^{\mathrm{XY}} \gamma_{31}^{\mathrm{Z}}\left(\lambda_{ \pm}-\lambda_{\mp}\right)}
\end{gathered}
$$

Here $\Sigma$ is the sum of all the rates and $D$ is a discriminant: $\uparrow$

$$
\begin{gathered}
\Sigma=k_{23}^{\mathrm{XY}}+k_{23}^{\mathrm{Z}}+\gamma_{31}^{\mathrm{XY}}+\gamma_{31}^{\mathrm{Z}} \\
D=\sqrt{\Sigma^{2}-4\left(k_{23}^{\mathrm{XY}} \gamma_{31}^{\mathrm{Z}}+k_{23}^{\mathrm{Z}} \gamma_{31}^{\mathrm{XY}}+\gamma_{31}^{\mathrm{XY}} \gamma_{31}^{\mathrm{Z}}\right)}
\end{gathered}
$$

The autocorrelation functions could be well fitted by a sum of two exponential functions with an offset:

$$
G^{(2)}(\tau)=c_{0}+c_{1} \exp \left(-\lambda_{1} \tau\right)+c_{2} \exp \left(-\lambda_{2} \tau\right)
$$

where the offset $c_{0}$ accounts for long-time residual correlations that are unrelated to the ISC. $c_{0}$ was generally close to 1 . The contrasts $\left(C_{1}=c_{1} / c_{0}\right.$ and $\left.C_{2}=c_{2} / c_{0}\right)$ were corrected for detector dark counts and stray light using the relation: ${ }^{6}$

$$
\left(G_{\text {theory }}^{(2)}(\tau)-1\right)=\left(1+\frac{\mathrm{B}}{\mathrm{S}}\right)^{2}\left(G_{\text {exp }}^{(2)}(\tau)-1\right)
$$

where the signal-to-background ratios (S/B) where determined from the fits of the excitation spectra.

$\S$ Note: in ref. 15, in the expression for $S$ (denoted $D$ in the present paper) in eqn (4) there is a typographical error: the parenthesized term inside the square brackets should be squared.

I $D$ is the discriminant of the second order polynomial obtained from the characteristic polynomial of the rate matrix after dividing out the zero at the origin corresponding to the steady-state solution.
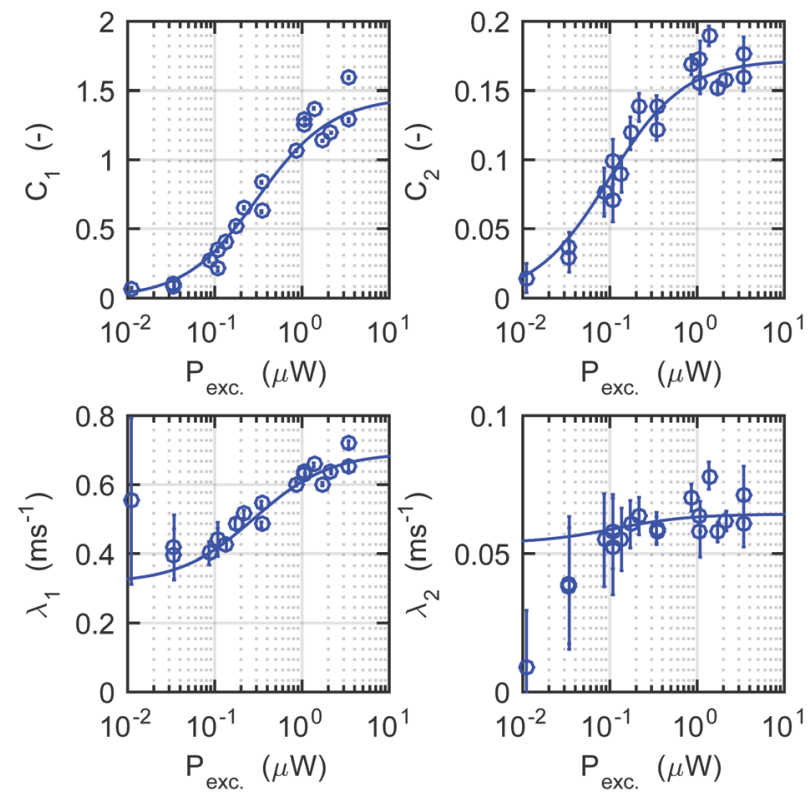

Fig. 4 The power dependence of the contrasts $C_{1}$ and $C_{2}$ and decay rates $\lambda_{1}$ and $\lambda_{2}$ of the autocorrelation function. The blue dots are the data points. The solid blue curves are a simultaneous error-weighted least-squares fit to the data in all four figures.

The excited state population $\tilde{\rho}_{22}$ and therefore the effective ISC rates $k_{23}^{\mathrm{XY}}$ and $k_{23}^{\mathrm{Z}}$ depend on the excitation power. By fitting eqn (2) and (3) to the decay rates and contrasts obtained from the autocorrelation functions at different excitation powers, we can deduce the ISC rates $\gamma_{23}^{\mathrm{XY}}, \gamma_{31}^{\mathrm{XY}}, \gamma_{23}^{\mathrm{Z}}$ and $\gamma_{31}^{\mathrm{Z}}$, see Fig. 4 . As was done previously for pentacene, ${ }^{2,3,5}$ terrylene ${ }^{9}$ and dibenzanthanthrene ${ }^{15}$ the slowly decaying component was attributed to the triplet state $\mathrm{T}_{\mathrm{Z}}$ while the fast decaying component was attributed to the states $T_{X}$ and $T_{Y}$. The results for 4 different perylene molecules are summarized in Table 1 . The rates to and from the triplet states have comparable orders of magnitude explaining the good contrast observed in the autocorrelation function.

The average phosphorescence lifetimes calculated from our measurements are $\left\langle\tau_{\mathrm{ph} .}^{\mathrm{XY}}\right\rangle=\left\langle\left(\gamma_{31}^{\mathrm{XY}}\right)^{-1}\right\rangle=3.0 \mathrm{~ms}$ and $\left\langle\tau_{\mathrm{ph} .}^{\mathrm{Z}}\right\rangle=$ $\left\langle\left(\gamma_{31}^{\mathrm{Z}}\right)^{-1}\right\rangle=19 \mathrm{~ms}$. These values are on the same orders of magnitude as the values that were reported in the literature previously, see Table 2. To the authors' best knowledge, this is however the first time two phosphorescence lifetimes are reported for perylene. Moreover the phosphorescence lifetime of $19 \mathrm{~ms}$, although on the same order of magnitude, is larger than any value reported previously in the literature.

Table 1 The ISC rates and homogeneous linewidth for 4 perylene molecules. The reported errors are the statistical errors calculated from the error weighted fits. The data shown in Fig. 2-4 is for molecule 3 in this table

\begin{tabular}{lcccc}
\hline & Molecule 1 & Molecule 2 & Molecule 3 & Molecule 4 \\
\hline$\gamma_{23}^{\mathrm{XY}} / \mathrm{ms}^{-1}$ & $0.74 \pm 0.04$ & $0.85 \pm 0.35$ & $0.72 \pm 0.03$ & $0.90 \pm 0.03$ \\
$\gamma_{31}^{\mathrm{XY}} / \mathrm{ms}^{-1}$ & $0.352 \pm 0.008$ & $0.36 \pm 0.02$ & $0.315 \pm 0.008$ & $0.311 \pm 0.007$ \\
$\gamma_{23}^{\mathrm{Z}} / \mathrm{s}^{-1}$ & $48 \pm 8$ & $49 \pm 24$ & $52 \pm 11$ & $62 \pm 9$ \\
$\gamma_{31}^{\mathrm{Z}} / \mathrm{s}^{-1}$ & $58 \pm 7$ & $53 \pm 12$ & $54 \pm 9$ & $52 \pm 7$ \\
$\Gamma_{\mathrm{hom}} / \mathrm{MHz}$ & $47 \pm 2$ & $46 \pm 3$ & $53 \pm 3$ & $40 \pm 1$
\end{tabular}


Table 2 Phosphorescence lifetimes of perylene previously reported in the literature. Our measurements gave $\left\langle\tau_{\mathrm{ph} .}^{\mathrm{XY}}\right\rangle=3.0 \mathrm{~ms}$ and $\left\langle\tau_{\mathrm{ph} .}^{\mathrm{Z}}\right\rangle=19 \mathrm{~ms}$. The lifetimes reported for perylene in polymethylmethacrylate were measured for protonated perylene $\left(\operatorname{Pr}-\mathrm{h}_{12}\right)$ and deuterated perylene $\left(\operatorname{Pr}-\mathrm{d}_{12}\right){ }^{29}$ As was pointed out in ref. 30, the lifetimes reported for perylene in acetonenitrile may have been shortened by the presence of oxygen

\begin{tabular}{llll}
\hline Ref. & Solvent & Conditions & $\tau_{\mathrm{ph} .} / \mathrm{ms}$ \\
\hline 23 & Anthracene & Bulk, 15 K & $13.0 \pm 0.1$ \\
29 & Polymethylmethacrylate & Bulk, 298 K, Pr-h ${ }_{12}$ & 6 \\
& & Bulk, 298 K, Pr-d 12 & 14 \\
31 & Ethanol & Bulk, 21 ${ }^{\circ} \mathrm{C}$ & 4.34 \\
& $n$-Hexane & & 4.84 \\
& Cyclohexane & Single mol., 1.7 K & 3.92 \\
22 & $n$-Nonane & Bulk, 300 K & 0.3 \\
32 and 30 & Acetonenitrile & &
\end{tabular}

One might expect an external heavy-atom effect due to the presence of chlorine atoms in the $o$ DCB host matrix. ${ }^{36,37}$ However if there is such an effect one would expect the phosphorescence lifetime to be shortened, instead the phosphorescence lifetime $\tau_{\mathrm{ph}}^{\mathrm{XY}}$ is comparable to the values reported for other host matrices and the value for $\tau_{\mathrm{ph}}^{\mathrm{Z}}$. is larger than reported for other host matrices (see Table 2). To draw any conclusions about a heavyatom effect would require more investigations, e.g. a comparison to perylene in other ortho-dihalogenated benzene host matrices (i.e. ortho-difluorobenzene, ortho-dibromobenzene and orthodiiodobenzene).

We did not measure the fluorescence lifetime of perylene, however if we assume the smallest measured homogeneous line width of $40 \mathrm{MHz}$ to be lifetime limited, we can estimate the fluorescence lifetime to be $\tau_{\mathrm{fl} .}=\left(2 \pi \Gamma_{\text {hom. }}\right)^{-1}=4.0 \mathrm{~ns}$. This value is probably a reasonable estimate: for perylene in ethanol and in a Shpols'kii matrix of $n$-octane a lifetime of $7 \pm 2$ ns was reported $^{38}$ for perylene in benzene fluorescence lifetimes of $4.79 \mathrm{~ns}^{39}$ and $5.02 \pm 0.1 \mathrm{~ns}^{40}$ were reported and for perylene in biphenyl a lifetime of $3.6 \mathrm{~ns}$ was reported. ${ }^{23}$ Using our estimate for the fluorescence lifetime we can make a rough estimate of the triplet yield $\phi_{\mathrm{T}}$. We find $\phi_{\mathrm{T}}=\tau_{\mathrm{fl}}\left(\gamma_{23}^{\mathrm{XY}}+\gamma_{23}^{\mathrm{Z}}\right)=3 \times 10^{-6}$.

\section{Conclusion}

By studying the photon bunching in the fluorescence from single perylene molecules in an oDCB host-matrix we were able to determine the ISC rates. In our measurements we could distinguish two out of three triplet states. The ISC rates from the excited singlet state $S_{1}$ to the triplet states $T_{X Y}$ and $T_{Z}$ were $0.80 \mathrm{~ms}^{-1}$ and $53 \mathrm{~s}^{-1}$ respectively. The average ISC rates from the triplet states $T_{X Y}$ and $T_{Z}$ to the ground state $S_{0}$ are $0.335 \mathrm{~ms}^{-1}$ and $54 \mathrm{~s}^{-1}$ respectively, which correspond well to phosphorescence lifetimes previously reported in literature. We estimate the triplet yield to be on the order of $3 \times 10^{-6}$.

Further experiments on perylene in oDCB could consist e.g. of optically detected magnetic resonance (ODMR) measurements to confirm the ISC rates and to determine the energy separation between the triplet states or phosphorescence spectroscopy to determine the energy of the singlet-triplet transition.

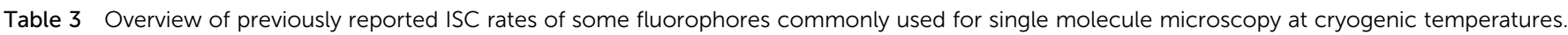

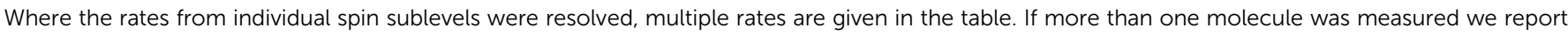
average values. At the end of the table we report the average rates that we obtained for perylene. RT: room temperature

\begin{tabular}{|c|c|c|c|c|c|c|}
\hline Guest/host & Ref. & $\gamma_{23} / \mathrm{s}^{-1}$ & & $\gamma_{31} / s^{-1}$ & & $T / \mathrm{K}$ \\
\hline \multicolumn{6}{|l|}{ Pentacene } & 1.8 \\
\hline /para-Terphenyl & 6 & $2.84 \times 10^{5}$ & & $2.24 \times 10^{4}$ & & 1.8 \\
\hline In-Tetradecane & 7 & $(5.2 \pm 1.5) \times 10^{5}$ & & $(2.1 \pm 0.2) \times 10^{4}$ & & 1.7 \\
\hline /Naphthalene & 8 & $1.2 \times 10^{6}$ & & $2.8 \times 10^{4}$ & & 1.4 \\
\hline \multicolumn{7}{|l|}{ Terrylene } \\
\hline /para-Terphenyl & 33 & $(5.40 \pm 0.17) \times 10^{2}$ & & $(2.96 \pm 0.03) \times 10^{3}$ & $(1.76 \pm 0.03) \times 10^{3}(3.17 \pm 0.09) \times 10^{2}$ & 1.8 \\
\hline /para-Terphenyl & 34 & $9.5 \times 10^{5}$ & & $6.9 \times 10^{3}$ & & RT \\
\hline /para-Terphenyl & 9 & $2.0 \times 10^{3}$ & $4.0 \times 10^{2}$ & $1.9 \times 10^{3}$ & $8 \times 10^{1}$ & 1.4 \\
\hline Ipara-Terphenyl & 10 & $(1.0 \pm 0.5) \times 10^{2}$ & & $(2.5 \pm 0.5) \times 10^{3}$ & & 5 \\
\hline /Anthracene & 11 and 12 & $(1.0 \pm 0.5) \times 10^{6}$ & & $(1.8 \pm 0.3) \times 10^{3}$ & & 1.5 \\
\hline /2,3-Dichloronaphthalene & 14 & $(1.62 \pm 0.49) \times 10^{4}$ & & $(6.0 \pm 0.4) \times 10^{3}$ & & 125 \\
\hline \multicolumn{7}{|l|}{ Dibenzanthanthrene } \\
\hline /n-Hexadecane & 15 & $1.25 \times 10^{3}$ & $4.0 \times 10^{1}$ & $4.50 \times 10^{3}$ & $7.50 \times 10^{2}$ & 1.8 \\
\hline /Naphthalene & 17 & $4.80 \times 10^{2}$ & $3.0 \times 10^{1}$ & $5.40 \times 10^{3}$ & $9.00 \times 10^{2}$ & 1.8 \\
\hline \multicolumn{7}{|l|}{ Terylenediimide } \\
\hline /Hexadecane & 18 & $(5 \pm 2) \times 10^{3}$ & $<10^{3}$ & $(1.5 \pm 0.1) \times 10^{4}$ & $(1.5 \pm 1) \times 10^{3}$ & 1.4 \\
\hline \multicolumn{7}{|l|}{ Perylene bisimide } \\
\hline /Hexadecane & 19 & $2.8 \times 10^{4}$ & $9.5 \times 10^{2}$ & $3.0 \times 10^{4}$ & $2.8 \times 10^{3}$ & 1.5 \\
\hline \multicolumn{7}{|l|}{ Dibenzoterrylene } \\
\hline /Anthracene & 12 & & & $2.5 \times 10^{4}$ & & 1.4 \\
\hline /Anthracene & 35 & & & $(1.4 \pm 0.6) \times 10^{4}$ & & $\mathrm{RT}$ \\
\hline $\begin{array}{l}\text { Perylene } \\
\text { /ortho-Dichlorobenzene }\end{array}$ & & $8.0 \times 10^{2}$ & $5.3 \times 10^{1}$ & $3.3 \times 10^{2}$ & $5.4 \times 10^{1}$ & 1.3 \\
\hline
\end{tabular}




\section{Acknowledgements}

We thank Peter Gast and Harmen van der Meer for their assistance with the cryogenics. This work is supported by NanoNextNL, a micro and nanotechnology consortium of the Government of the Netherlands and 130 partners. This work is part of the research programme of the Foundation for Fundamental Research on Matter (FOM), which is part of the Netherlands Organization for Scientific Research (NWO).

\section{References}

1 M. Orrit, Nature, 2009, 460, 42-44.

2 J. Köhler, J. Disselhorst, M. Donckers, E. Groenen, J. Schmidt and W. Moerner, Nature, 1993, 363, 242-244.

3 J. Wrachtrup, C. von Borczyskowski, J. Bernard, M. Orrit and R. Brown, Nature, 1993, 363, 244-245.

4 J. Wrachtrup, C. von Borczyskowski, J. Bernard, M. Orrit and R. Brown, Phys. Rev. Lett., 1993, 71, 3565-3568.

5 R. Brown, J. Wrachtrup, M. Orrit, J. Bernard and C. von Borczyskowski, J. Chem. Phys., 1994, 100, 7182-7191.

6 J. Bernard, L. Fleury, H. Talon and M. Orrit, J. Chem. Phys., 1993, 98, 850-859.

7 B. Kozankiewicz, M. Banasiewicz, J. Dresner and M. Orrit, Chem. Phys. Lett., 2001, 343, 71-76.

8 S. Kummer, C. Bräuchle and T. Basché, Mol. Cryst. Liq. Cryst., 1996, 283, 255-260.

9 S. Kummer, T. Basché and C. Bräuchle, Chem. Phys. Lett., 1994, 229, 309-316.

10 M. Banasiewicz, O. Morawski, D. Wiacek and B. Kozankiewicz, Chem. Phys. Lett., 2005, 414, 374-377.

11 A. Nicolet, M. Kol'chenko, B. Kozankiewicz and M. Orrit, J. Chem. Phys., 2006, 124, 164711.

12 A. Nicolet, PhD thesis, Leiden University, Leiden, The Netherlands, 2007.

13 M. Kol'chenko, B. Kozankiewicz, A. Nicolet and M. Orrit, Opt. Spectra, 2005, 98, 745-751.

14 M. Białkowska, W. Chaładaj, A. Makarewicz and B. Kozankiewicz, Acta Phys. Pol., A, 2015, 128, RK.128.3.1.

15 A. Boiron, B. Lounis and M. Orrit, J. Chem. Phys., 1996, 105, 3969-3974.

16 F. Jelezko, B. Lounis and M. Orrit, J. Chem. Phys., 1997, 107, 1692-1702.

17 F. Zhelezko, I. Gulis, B. Lounis and M. Orrit, J. Appl. Spectrosc., 1999, 66, 344-352.
18 A. Mais, J. Tittel, T. Basché, C. Bräuchle, W. Göhde, H. Fuchs, G. Müller and K. Müllen, J. Phys. Chem. A, 1997, 101, 8435-8440.

19 E. Lang, F. Würthner and J. Köhler, ChemPhysChem, 2005, 6, 935-941.

20 E. Lang, R. Hildner, H. Engelke, P. Osswald, F. Würthner and J. Köhler, ChemPhysChem, 2007, 8, 1487-1496.

21 T. Basché and W. Moerner, Nature, 1992, 355, 335-337.

22 M. Pirotta, A. Renn, M. Werts and U. Wild, Chem. Phys. Lett., 1996, 250, 576-582.

23 P. Walla, F. Jelezko, P. Tamarat, B. Lounis and M. Orrit, Chem. Phys., 1998, 233, 117-125.

24 A. Shimoda, T. Hikida and Y. Mori, J. Phys. Chem., 1979, 83, 1309-1312.

25 T. Ichimura, K. Nahara and Y. Mori, J. Photochem., 1986, 33, 49-60.

26 A. A. Gorshelev, A. V. Naumov, I. Y. Eremchev, Y. G. Vainer, L. Kador and J. Köhler, ChemPhysChem, 2010, 11, 182-187.

27 P. Navarro Pérez, PhD thesis, Leiden University, Leiden, The Netherlands, 2014.

28 R. Boese, M. Kirchner, J. Dunitz, G. Filippini and A. Gavezzotti, Helv. Chim. Acta, 2001, 84, 1561-1577.

29 R. Kellogg and N. Convers Wyeth, J. Chem. Phys., 1966, 45, 3156-3158.

30 T. Sander, H. Löhmannsröben and H. Langhals, J. Photochem. Photobiol., A, 1995, 86, 103-108.

31 C. Parker and A. Thelma, Chem. Commun., 1966, 108b-109.

32 M. Komfort, H. Löhmannsröben and T. Salthammer, J. Photochem. Photobiol., A, 1990, 51, 215-227.

33 A. Brouwer, E. Groenen and J. Schmidt, Phys. Rev. Lett., 1998, 80, 3944-3947.

34 L. Fleury, J.-M. Segura, G. Zumofen, B. Hecht and U. Wild, Phys. Rev. Lett., 2000, 84, 1148-1151.

35 K. D. Major, PhD thesis, Imperial College London, London, United Kingdom, 2016.

36 S. McGlynn, M. Reynolds, G. Daigre and N. Christodoyleas, J. Phys. Chem., 1962, 66, 2499-2505.

37 S. McGlynn, J. Daigre and F. Smith, J. Chem. Phys., 1963, 39, 675-679.

38 I. Abram, R. Auerbach, R. Birge, B. Kohler and J. Stevenson, J. Chem. Phys., 1975, 63, 2473-2478.

39 S. Strickler and R. A. Berg, J. Chem. Phys., 1962, 37, 814-822.

40 W. R. Ware and B. A. Baldwin, J. Chem. Phys., 1964, 40, 1703-1705. 